\title{
Three thermodynamically-based parametrizations of the deceleration parameter
}

\author{
Sergio del Campda, I Ivan Duranb,2 Ramón Herrerad, ${ }^{1}$ and Diego Pavónd2 \\ ${ }^{1}$ Instituto de Física, Pontificia Universidad Católica de Valparaíso, \\ Av. Universidad 330, Campus Curauma, Valparaíso, Chile \\ ${ }^{2}$ Departamento de Física, Facultad de Ciencias, \\ Universidad Autónoma de Barcelona, \\ 08193 Bellaterra (Barcelona), Spain
}

\begin{abstract}
We propose, and constrain with the latest observational data, three model-independent parametrizations of the cosmic deceleration parameter $q(z)$. They are well behaved and stay finite at all redshifts. We construct them by fixing the value of $q$ at high redshift, $q(z \gg 1)=1 / 2$ (as demanded by cosmic structure formation), and at the far future, $q(z=-1)=-1$, and smoothly interpolating $q(z)$ between them. The fixed point at $z=-1$ is not arbitrarily chosen; it readily follows from the second law of thermodynamics. This fairly reduces the ample latitude in parameterizing $q(z)$.
\end{abstract}

\footnotetext{
${ }^{\mathrm{a}}$ E-mail: sdelcamp@ucv.cl

b E-mail: ivan.duran@uab.cat

c E-mail: ramon.herrera@ucv.cl

d E-mail: diego.pavon@uab.es
} 


\section{INTRODUCTION}

The deceleration parameter, defined as $q=-1-\left(\dot{H} / H^{2}\right)$, is a key quantity in describing the evolution of the homogeneous and isotropic universe. Its importance lies in the fact that it tells us the rate at which the Universe accelerates or decelerates its expansion. (Here and throughout $H=\dot{a} / a$ denotes the Hubble function, and $a$ the scale factor of the FriedmannLemaître-Robertson-Walker (FLRW) metric). Unfortunately, at present measurements of $q$ suffer from non-small uncertainties that quickly grow with redshift $\left(z=a^{-1}-1\right.$ with $\left.a_{0}=1\right)$, though it is virtually certain that the Universe is accelerating nowadays, i.e., that $q_{0}<0$ (the zero subscript means present time). Expressions of $q(z)$ provided by cosmological models are of not much help either because none of them rests on sufficiently convincing theoretical grounds. It is to be hoped that things will eventually turn for the better when a successful theory of quantum gravity is in place, though it may well take a long while.

Nevertheless, on the observational side the situation may improve comparatively soon given the variety and range of ongoing and planned major ambitious projects that involve bigger telescopes and advanced techniques -for a short review and a comprehensive list of references see $\S 14$ of [1]. In view of the above, it seems reasonable to propose parametrized expressions of $q(z)$ based not in any specific cosmological model but on practical and empirical reasons that lessen their wide latitude. They can be useful while we patiently wait for a theoretically sound model backed by observation at all scales.

Thus far, different parametrizations, such as $q=q_{0}+q_{1} z, q=q_{0}+q_{1} z(1+z)^{-1}$, $q=q_{1}+q_{2} z(1+z)^{-2}, q=1 / 2+q_{1}(1+z)^{-2}, q=1 / 2+\left(q_{1} z+q_{2}\right)(1+z)^{-2}$, and more complex than these, have been considered in the literature to reconstruct $q(z)$ from observational data (see e.g. [2]-[8]). However, the first parametrization is adequate for $|z| \ll 1$ only and the others are unsuitable to predict the behavior of the deceleration parameter in the far future; in particular, they diverge as $z \rightarrow-1$. Parametrizations whose intended range of validity includes the far future are necessarily more involved and usually contain three or four free parameters [9, 10].

In this paper we propose three model independent parametrizations, with just two free parameters, valid from the matter dominated epoch $(z \gg 1)$ onwards (i.e., up to $z=-1$ ), based on practical and theoretical reasons and independent of any cosmological model. By construction they obey the asymptotic conditions, $q(z \gg 1)=1 / 2, q(z=-1)=-1$, and 
a further condition, $d q / d z>0$, which is valid at least when $q \rightarrow-1$. The first condition expresses the conviction that at sufficiently high redshift the Universe was matter dominated (otherwise it would be very hard to account for the observed cosmic structures). At first sight, the other conditions are less compelling. As explained below, they are based on the second law of thermodynamics when account is made of the entropy of the apparent horizon. Usually one parametrizes a function in any specific interval by interpolating it between two given points (one at either end of the interval), modulo one first knows the value taken by the function at these two points. In actual fact, the parametrizations of $q(z)$ proposed so far have just one fixed point: the asymptotic value at high redshift $(q$ must converge to $1 / 2$ when $z \gg 1$ ). The other, $q_{0}$, is not in reality a fixed point because the value of the deceleration parameter at $z=0$ is not very well known and therefore left free. The parametrizations proposed in this paper have two fixed points, one at the far past $(z \gg 1)$, and other at the far future $(z=-1)$. The second fixed point conforms to the thermodynamical constraints imposed by the second law. We believe this means a clear advantage over previous parametrizations of $q(z)$, with just one fixed point. While it can be found in the literature parameterizations that also fix $q$ at $z=-1$ they do so arbitrarily, i.e., not grounded on sound physics.

The aim of this paper is to propose three model-independent parametrizations of $q(z)$, from $z \gg 1$ up to $z=-1$, that comply with the second law of thermodynamics and constrain their two free parameters with recent observational data. As it turns out, all of them predict that the present stage of accelerated expansion will never slow down and are consistent with the spatially flat $\Lambda$ CDM model.

Section [I] considers the properties the deceleration parameter must observe assuming the Universe obeys the second law of thermodynamics. Put otherwise if, in the long run, it tends to an equilibrium state, i.e., of maximum entropy. Section III presents the statistical tools, to be employed later, that make use of observational data from supernovae type Ia (557 data points), baryon acoustic oscillations combined with cosmic microwave background (BAO/CMB) (7 data points), and the history of the Hubble factor (24 data points). Section IV presents the three parametrizations, constrain them with the observational data, and discuss them. Finally, Section $[\mathrm{V}]$ summarizes our work and introduces some final remarks. 


\section{THERMODYNAMICAL CONSTRAINTS ON $q(z)$}

As is well known, physical systems tend spontaneously to some equilibrium state compatible with the constraints imposed on them. This summarizes the empirical basis of the second law of thermodynamics. Very briefly, this law establishes that isolated, macroscopic systems, evolve to the maximum entropy state consistent with their constraints [11]. As a consequence their entropy, $S$, cannot decrease at any time, i.e., $d S \geq 0$. Further, in the last phase of the evolution $S$ has to be a convex function of the said variable, $d^{2} S(z \rightarrow-1)<0$.

Arguably, the entropy of Friedmann-Lemaittre-Robertson-Walker (FLRW) universes is dominated by the entropy of the causal horizon, at least at late times -see e.g. [12]. As causal horizon we shall take the apparent horizon, the marginally trapped surface with vanishing expansion of radius $\tilde{r}_{A}=1 / \sqrt{H^{2}+k a^{-2}}$ [13], where $k$ denotes the spatial curvature index. Interestingly enough, it has been shown that this horizon represents the appropriate thermodynamic boundary surface [14]. Leaving aside possible quantum corrections its entropy results proportional to area of the latter $\left(4 \pi \tilde{r}_{A}^{2}\right)[13]$,

$$
S_{A} \propto \mathcal{A}=4 \pi\left(H^{2}+k a^{-2}\right)^{-1}
$$

Therefore, so long as we can ignore the entropy within the horizon, the second law of

thermodynamics imposes $\mathcal{A}^{\prime} \geq 0$, at any time, as well as $\mathcal{A}^{\prime \prime} \leq 0$ at late times -the prime meaning derivative with respect to the scale factor. Both conditions are to be fulfilled if the FLRW universe is to tend to thermodynamic equilibrium at late times [15].

Bearing in mind the definition of the deceleration parameter, for spatially flat $(k=0)$ FLRW universes we can write

$$
\mathcal{A}^{\prime}=2 \mathcal{A} \frac{1+q}{a}, \quad \text { and } \quad \mathcal{A}^{\prime \prime}=2 \mathcal{A}\left[2\left(\frac{1+q}{a}\right)^{2}+\frac{q^{\prime}}{a}-2 \frac{1+q}{a^{2}}\right] \text {. }
$$

The first equation implies $q \geq-1$. Inspection of the second one reveals that when $a \rightarrow \infty$ the middle term in the square parenthesis dominates. Thereby, $d q / d a<0$ in that limit. Thus, for the Universe to tend to thermodynamic equilibrium at late times we must have $q \rightarrow-1$ and $d q / d z>0$ as $z \rightarrow-1$. 


\section{STATISTICAL TOOLS}

This Section outlines the use of the observational data to fit the parametrizations of the deceleration parameter, $q(z)$. Since the likelihood function is defined by $\mathcal{L} \propto \exp \left(-\chi^{2} / 2\right)$ the best fit to the data follows from minimizing the sum $\chi_{\text {total }}^{2}=\chi_{S N}^{2}+\chi_{B A O / C M B}^{2}+\chi_{H}^{2}$. As detailed below, the best fit values of the parameters can be obtained by contrasting the proposed parametrizations with the empirical data mentioned above and minimizing the $\chi_{\text {total }}^{2}$ by means of the Markov Chain Monte Carlo method.

\section{A. SN Ia}

We compare the theoretical distance modulus

$$
\mu_{t h}\left(z_{i}\right)=5 \log _{10}\left(\frac{d_{L}}{10 \mathrm{pc}}\right)+\mu_{0},
$$

where $\mu_{0}=42.38-5 \log _{10} h$, with the observed distance modulus $\mu_{\text {obs }}\left(z_{i}\right)$ of the $557 \mathrm{su}$ pernovae type Ia assembled in the Union2 compilation [16]. The latter data set is substantially richer than previous SN Ia compilations and presents other advantages; mainly, the refitting of all light curves with the SALT2 fitter and an upgraded control of systematic errors. In (3) $d_{L}=(1+z) \int_{0}^{z} \frac{d \tilde{z}}{E(\tilde{z} ; \mathbf{p})}$ is the Hubble-free luminosity distance, $\mathbf{p} \equiv\left(q_{1}, q_{2}\right)$, and $E(z ; \mathbf{p})=H(z ; \mathbf{p}) / H_{0}$. Here and throughout, $q_{1}$ and $q_{2}$ denote the free parameters occurring in the parametrizations presented below in Section IV; $H_{0}$ is the Hubble constant and $h$ its value in units of $100 \mathrm{~km} / \mathrm{s} / \mathrm{Mpc}$.

The $\chi^{2}$ from the $557 \mathrm{SN}$ Ia is given by

$$
\chi_{S N}^{2}(\mathbf{p})=\sum_{i=1}^{557} \frac{\left[\mu_{t h}\left(z_{i}\right)-\mu_{o b s}\left(z_{i}\right)\right]^{2}}{\sigma^{2}\left(z_{i}\right)},
$$

where the subscripts "th" and "obs" indicate the theoretical value (i.e., the value from the parametrization) and the observed value, respectively. As usual, the $\sigma_{i}$ quantities stand for the $1 \sigma$ uncertainty associated to the $i$ th data point. To eliminate the effect of the nuisance parameter $\mu_{0}$, which is independent of the data points and the data set, we follow the procedure of [17]. 


\section{B. BAO and CMB}

Baryon acoustic oscillations can be traced to pressure waves at the recombination epoch generated by cosmological perturbations in the primeval baryon-photon plasma. They have been revealed by a distinct peak in the large scale correlation function measured from the luminous red galaxies sample of the Sloan Digital Sky Survey (SDSS): at $z=0.35$ [18], as well as in the two degree Field Galaxy Redshift Survey at $z=0.2$ [19]. More recently other peaks have been observed: at $z=0.278$ (with the SDSS [20]), at $z=0.106$ (in the six degree Field Galaxy Redshift Survey [21]), and at $z=0.44, z=0.60$, and $z=0.73$ (by the WiggleZ team [22]).

From each peak the ratio of the comoving sound horizon $r_{s}(z)=\int_{z}^{\infty} c_{s}(z) / H(z) d z$ at decoupling $\left(z=z_{\star} \simeq 1090\right)$ and at the drag epoch $\left(z=z_{d}\right)$, the epoch at which the acoustic oscillations are frozen in, can be measured. Here $c_{s}$ is the speed of sound. Likewise, at each peak, a characteristic distance scale, the dilation scale

$$
D_{V}\left(z_{B A O}\right)=\left[z_{B A O} \frac{d_{A}^{2}\left(z_{B A O}\right)}{H\left(z_{B A O}\right)}\right]^{\frac{1}{3}}
$$

where $d_{A}(z)=\int_{0}^{z} \frac{d z^{\prime}}{H\left(z^{\prime}\right)}$ is the comoving angular distance, can also be determined. To compute the drag epoch redshift we use the formula (4) of Eisenstein and $\mathrm{Hu}$ in [23] and get $z_{d} \approx 1020$.

Multiplying the ratio, $\frac{r_{s}\left(z_{d}\right)}{D_{V}\left(z_{B A O}\right)}$, taken from the BAO peaks by the acoustic scale

$$
l_{A}=\pi \frac{d_{A}\left(z_{\star}\right)}{r_{s}\left(z_{\star}\right)}
$$

we get $\frac{d_{A}\left(z_{\star}\right)}{D_{V}\left(z_{B A O}\right)} \frac{r_{s}\left(z_{d}\right)}{r_{s}\left(z_{\star}\right)}$ at each redshift of the seven BAO data. Here we use the value for $l_{A}$ derived from Wilkinson microwave anisotropy probe (WMAP)7-years data, namely, $l_{A}=$ $302.09 \pm 0.76$ [24]. If we also use the value of the ratio of sound horizon at the drag epoch and at recombination (redshift $z_{\star}$ ), computed from the values reported in $[24] \frac{r_{s}\left(z_{d}\right)}{r_{s}\left(z_{\star}\right)}=$ $1.045 \pm 0.015$ we obtain the new estimator $\frac{d_{A}\left(z_{\star}\right)}{D_{V}\left(z_{B A O}\right)}$, shown in table [, as done in [25]. Using this estimator, the dependence in the sound horizons at decoupling and the drag epoch is suppressed. Thus we just use the ratio between them, which is almost model independent. This follows because both redshifts are rather close and the sound horizon at decoupling and drag essentially depend on the fractional difference between the number of photons and baryons [25]. To obtain the $\chi^{2}$ for the combined BAO/CMB data we compute 


\begin{tabular}{|c|c|c|c|c|c|c|c|}
\hline$z_{B A O}$ & 0.106 & 0.2 & 0.278 & 0.35 & 0.44 & 0.6 & 0.73 \\
\hline$\frac{r_{s}\left(z_{d}\right)}{D_{V}\left(z_{B A O}\right)}$ & $0.336 \pm 0.015$ & $0.1905 \pm 0.0061$ & $0.1394 \pm 0.0049$ & $0.1097 \pm 0.0036$ & $0.0916 \pm 0.0071$ & $0.0726 \pm 0.034$ & $0.0592 \pm 0.0032$ \\
\hline$\frac{d_{A}\left(z_{\star}\right)}{D_{V}\left(z_{B A O}\right)}$ & $30.92 \pm 1.45$ & $17.53 \pm 0.62$ & $12.83 \pm 0.49$ & $10.09 \pm 0.36$ & $8.43 \pm 0.66$ & $6.68 \pm 0.33$ & $5.45 \pm 0.30$ \\
\hline
\end{tabular}

Table I. Values of $\frac{r_{s}\left(z_{d}\right)}{D_{V}\left(z_{B A O}\right)}$ (reported in [18-22]) and the derived ratio $\frac{d_{A}\left(z_{\star}\right)}{D_{V}\left(z_{B A O}\right)}$.

$$
\chi_{B A O / C M B}^{2}=\mathbf{X}^{T} \mathbf{C}^{-1} \mathbf{X}
$$

where

$$
\mathbf{X}=\left(\begin{array}{c}
\frac{d_{A}\left(z_{\star}\right)}{D_{V}(0.106)}-30.92 \\
\frac{d_{A}\left(z_{\star}\right)}{D_{V}(0.2)}-17.53 \\
\frac{d_{A}\left(z_{\star}\right)}{D_{V}(0.278)}-12.83 \\
\frac{d_{A}\left(z_{\star}\right)}{D_{V}(0.35)}-10.09 \\
\frac{d_{A}\left(z_{\star}\right)}{D_{V}(0.44)}-8.43 \\
\frac{d_{A}\left(z_{\star}\right)}{D_{V}(0.6)}-6.68 \\
\frac{d_{A}\left(z_{\star}\right)}{D_{V}(0.73)}-5.45
\end{array}\right)
$$

and $\mathbf{X}^{T}$ the transpose matrix. The elements of covariance matrix $\mathbf{C}$ are given by

$$
\mathbf{C}_{i j}=\sum_{k}\left(\frac{\partial \frac{d_{A}\left(z_{\star}\right)}{D_{V}(z)}}{\partial p_{k}}\right)_{z_{i}}\left(\frac{\partial \frac{d_{A}\left(z_{\star}\right)}{D_{V}(z)}}{\partial p_{k}}\right)_{z_{j}} \mathbf{C}_{p_{k} i j}
$$

where the sum is over the estimators used (in our case, $\frac{r_{s}\left(z_{d}\right)}{D_{V}\left(z_{B A O}\right)}, l_{A} / \pi$ and $\frac{r_{s}\left(z_{d}\right)}{r_{s}\left(z_{\star}\right)}$ ). The elements of the original covariance matrices are $\mathbf{C}_{\frac{l_{A}}{\pi}}=\theta_{\frac{l_{A}}{\pi}}^{2}, \mathbf{C}_{\frac{r_{s}\left(z_{d}\right)}{r_{s}\left(z_{\star}\right)}}=\theta_{\frac{r_{s}\left(z_{d}\right)}{r_{s}\left(z_{\star}\right)}}^{2}$ and $\mathbf{C}_{B A O i j}=$ $\theta_{B A O}{ }_{i} \theta_{B A O}{ }_{j} r_{i j}$ where $\theta_{B A O} i$ stand for the errors associated with the estimator $\frac{r_{s}\left(z_{d}\right)}{D_{V}\left(z_{i}\right)}$. The only non-zero off-diagonal correlation coefficients $r_{i j}$ are $r_{z=0.2} z=0.35=0.337, r_{z=0.44} z=0.6=$ 0.369 and $r_{z=0.6} z=0.73=0.438$, and their symmetric [19, 22]. Thus, the inverse covariance matrix comes to be

$$
\mathbf{C}^{-1}=\left(\begin{array}{cccccccc}
0.492 & -0.084 & -0.126 & -0.136 & -0.025 & -0.081 & -0.088 \\
-0.084 & 3.362 & -0.327 & -2.397 & -0.065 & -0.209 & -0.228 \\
-0.126 & -0.327 & 4.429 & -0.528 & -0.098 & -0.314 & -0.342 \\
-0.136 & -2.397 & -0.528 & 9.712 & -0.106 & -0.338 & -0.368 \\
-0.025 & -0.065 & -0.098 & -0.106 & 2.798 & -2.749 & 1.182 \\
-0.081 & -0.209 & -0.314 & -0.338 & -2.749 & 15.002 & -7.294 \\
-0.088 & -0.228 & -0.342 & -0.368 & 1.182 & -7.294 & 14.587
\end{array}\right) .
$$




\section{History of the Hubble parameter}

The history of the Hubble parameter, $H(z)$, is poorly constrained though, recently, some high precision measurements by Riess et al. at $z=0$, obtained from the observation of 240 Cepheid variables of rather similar periods and metallicities [26], and Gaztañaga et al. at $z=0.24,0.34$, and 0.43 [27], who used the BAO peak position as a standard ruler in the radial direction, have improved matters somewhat. We have employed these four data alongside 11 less precise data, in the redshift interval $0.1 \lesssim z \lesssim 1.8$, from Simon et al.

[28] and Stern et al. [29], derived from the differential ages of passive-evolving galaxies and archival data. In addition we have included in our analysis 9 more recent correlated data from the WiggleZ survey [30].

The corresponding $\chi^{2}$

$$
\chi_{H}^{2}(\mathbf{p})=\sum_{i=1}^{15} \frac{\left[H_{t h}\left(z_{i}\right)-H_{o b s}\left(z_{i}\right)\right]^{2}}{\sigma^{2}\left(z_{i}\right)}+\mathbf{X}_{H}^{T} \mathbf{C}_{H}^{-1} \mathbf{X}_{H},
$$

where

$$
\mathbf{X}_{H}=\left(\begin{array}{c}
H_{t h}(0.05)-69.4 \\
H_{t h}(0.15)-76.6 \\
H_{t h}(0.25)-75.3 \\
H_{t h}(0.35)-78.3 \\
H_{t h}(0.45)-87.3 \\
H_{t h}(0.55)-88.9 \\
H_{t h}(0.65)-101.4 \\
H_{t h}(0.75)-96.9 \\
H_{t h}(0.85)-127.3
\end{array}\right)
$$

and $\mathbf{C}_{H}^{-1}$ is the inverse covariance matrix given in table 6 of [30].

\section{PARAMETRIZATIONS}

Here we propose and constrain three parametrizations of the deceleration parameter, valid from the matter dominated era up to $z=-1$. These fulfill: $(i) q(z \gg 1)=1 / 2$ (as demanded by cosmic structure formation), (ii) $q(z=-1)=-1$ and $d q(z) / d z>0$ when $q(z) \rightarrow-1$ as required by the thermodynamic arguments of above (the second law of 
thermodynamics). In interpolating between $(z=-1, q=-1)$ and $(z \gg 1, q=1 / 2)$ we introduce two free parameters $q_{1}$ and $q_{2}$ and fit them to the observational sets of data by the method of last Section. Note that due to the scarcity of $q(z)$ data and their big error bars, we do not constrain the parametrizations directly. We constrain instead the expressions for $H(z)$ that arise from integrating them; namely,

$$
H(z)=H_{0} \exp \left\{\int_{0}^{z}[1+q(x)] d \ln (1+x)\right\},
$$

which holds for all parametrizations. This has the advantage of a much bigger and robust statistics. Notice that the Hubble constant also enters this expression as a free parameter. Its value for each parametrization is obtained by fitting it to the $H(z)$ data, [26 30] .

\section{A. Parametrization I}

As a first parametrization we propose

$$
q(z)=-1+\frac{3}{2}\left(\frac{(1+z)^{q_{2}}}{q_{1}+(1+z)^{q_{2}}}\right),
$$

where to avoid divergences $q_{1}$ and $q_{2}$ must be positive-definite.

Introducing (11) in (10), it follows

$$
H(z)=H_{0}\left(\frac{q_{1}+(1+z)^{q_{2}}}{q_{1}+1}\right)^{\frac{3}{2 q_{2}}} .
$$

(We note in passing that for $q_{2}=3$ the $\Lambda$ CDM behavior is reproduced). By using the method outlined in last section in conjunction with the observational data (SN Ia (557), $\mathrm{CMB} / \mathrm{BAO}(7)$ and $H(z)(15)$ ), we fit the three free parameters occurring in (12). The result is $q_{1}=2.87_{-0.53}^{+0.70}, q_{2}=3.27 \pm 0.55$, and $H_{0}=70.5_{-1.6}^{+1.5} \mathrm{~km} / \mathrm{s} / \mathrm{Mpc}$. Table II shows the $\chi^{2}$ values of the best fit.

\begin{tabular}{||l||lllll||}
\hline \hline Data sets & $\chi_{S N}^{2}$ & $\chi_{B A O / C M B}^{2}$ & $\chi_{H}^{2}$ & $\chi_{\text {tot }}^{2}$ & $\chi_{\text {tot }}^{2} /$ dof \\
\hline \hline Union2+BAO/CMB+Hubble & 542.6 & 2.6 & 17.9 & 563.3 & 0.96 \\
\hline \hline
\end{tabular}

Table II. Best fit $\chi^{2}$ values of parametrization I, Eq. (11). The free parameters are $q_{1}, q_{2}$ and $H_{0}$.

Figure 1 shows the evolution of the $q$ for the best fit values of parametrization I (solid line), with its $1 \sigma$ confidence region (shadowed area), and the spatially flat $\Lambda$ CDM model 
(dashed line) as determined by the WMAP 7-years team [24] (the latter graph is included for the sake of comparison), in the interval $-1 \leq z \leq 5$ (left panel), and the evolution of the Hubble function in the interval $0 \leq z \leq 3$ (right panel).
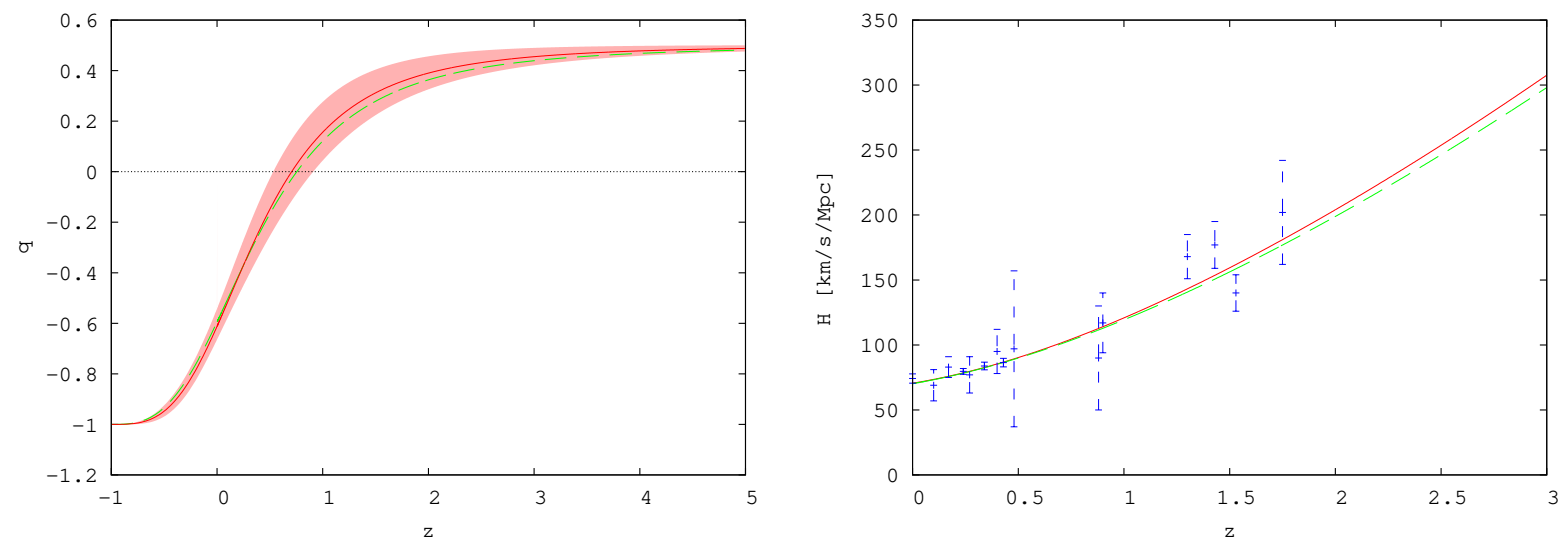

Figure 1. Left panel: deceleration parameter vs. redshift. The shaded area shows the $1 \sigma$ confidence region. Right panel: Hubble function vs. redshift (the observational data are borrowed from Refs. [26 29]). In both panels the solid (red) and the dashed (green) lines are used for the best fit of parametrization I (Eq. (11)) and for the $\Lambda$ CDM model with $\Omega_{M 0}=0.27$ and $H_{0}=72.1 \mathrm{~km} / \mathrm{s} / \mathrm{Mpc}$ -see [24]-, respectively. The latter graph is shown for comparison.

Figure 2 depicts the $1 \sigma$ and $2 \sigma$ contour plots of the pairs $\left(q_{1}, q_{2}\right)$ (left panel) and $\left(H_{0}\right.$, $q_{0}$ ) (right panel). Upon assuming that the expansion is dominated by pressureless matter and some other (unspecified) component, non-interacting between each other except gravitationally, the effective equation of state (EoS) parameter is given by

$$
w(z)=\frac{2 q(z)-1}{3\left(1-\Omega_{M}(z)\right)} .
$$

Parametrizations of $q(z)$ and $w(z)$ are somewhat equivalent but not quite because in the latter case some assumptions about the energy budget of the Universe, as well on the existence or not of possible interactions between the different components, have to be made while in the former (as in our case) not necessarily.

Having said this, it is interesting to confront (13) with the widely used ChevallierPolarsky-Linder (CPL) parametrization [31, 32]

$$
w=w_{0}+w_{1} \frac{z}{1+z}
$$



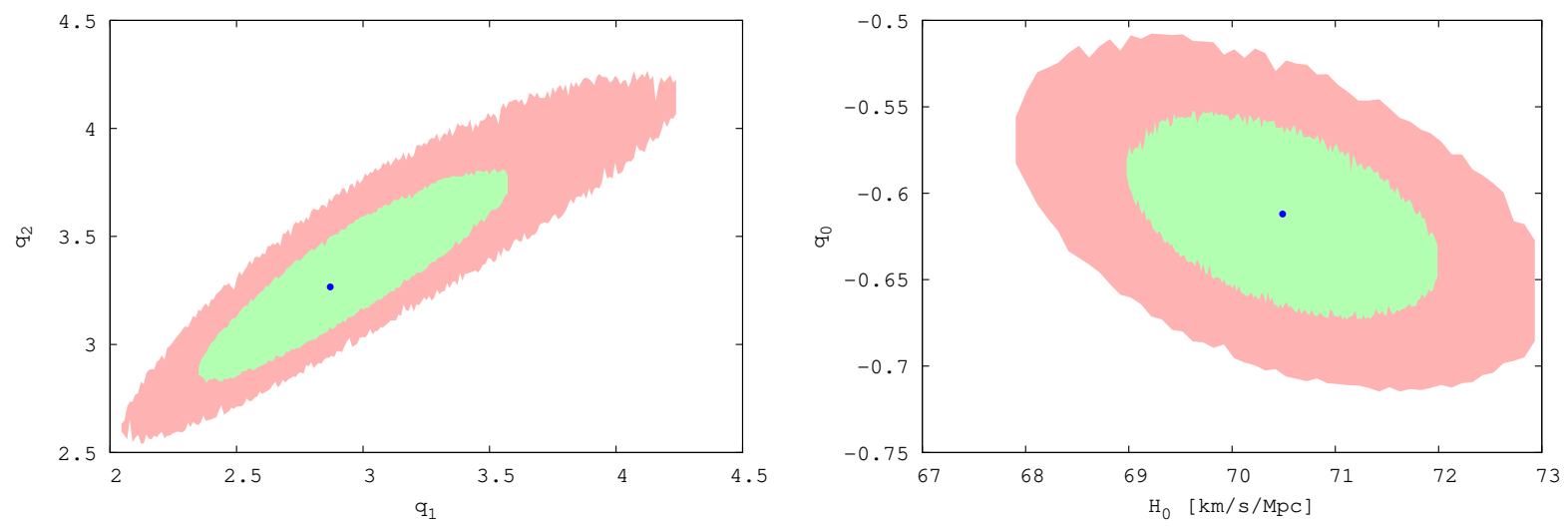

Figure 2. Left panel: $1 \sigma$ and $2 \sigma$ confidence regions of the pair of free parameters $\left(q_{1}, q_{2}\right)$ of parametrization I, Eq. (11). Right panel: $1 \sigma$ and $2 \sigma$ regions of the pair of free parameters $\left(H_{0}, q_{0}\right)$. The dot signals the best fit values.

not far from $z=0$, in the redshift range $-0.3 \leq z \leq 0.3$. We restrict ourselves to comparative small redshift around $z=0$ because it diverges at $z \rightarrow-1$. After numerically linearizing our expression for $w(z)$ we get $w_{0}=-0.92 \pm 0.10$ and $w_{1}=0.31_{-0.25}^{+0.28}$, values in very good agreement with those reported in [24], for the CPL parameters, namely: $w_{0}=-0.93 \pm 0.12$ and $w_{1}=-0.38_{-0.65}^{+0.66}$.

As Fig. 3 reveals, comparison in the extended interval $-0.5 \leq z \leq 5$ shows that for $z \geq 2.5$ the evolution of the effective of both EoS disagree in excess of $1 \sigma$. This is consistent with claims that the CPL parametrization is not appropriate to fit data simultaneously at low and high redshifts [33, 34].

\section{B. Parametrization II}

As a second parametrization we propose,

$$
q(z)=-\frac{1}{4}\left(3 q_{1}+1-3\left(q_{1}+1\right) \frac{q_{1} e^{q_{2}(1+z)}-e^{-q_{2}(1+z)}}{q_{1} e^{q_{2}(1+z)}+e^{-q_{2}(1+z)}}\right) .
$$

In this case the Hubble function must be obtained by numerically integrating Eq. (10). Proceeding as before we obtain, $q_{1}=0.078_{-0.043}^{+0.086}, q_{2}=0.95_{-0.20}^{+0.23}$, and $H_{0}=70.4 \pm 1.6$ $\mathrm{km} / \mathrm{s} / \mathrm{Mpc}$ for the three parameters entering $H(z)$. The $\chi^{2}$ best fit values are shown in table III. 


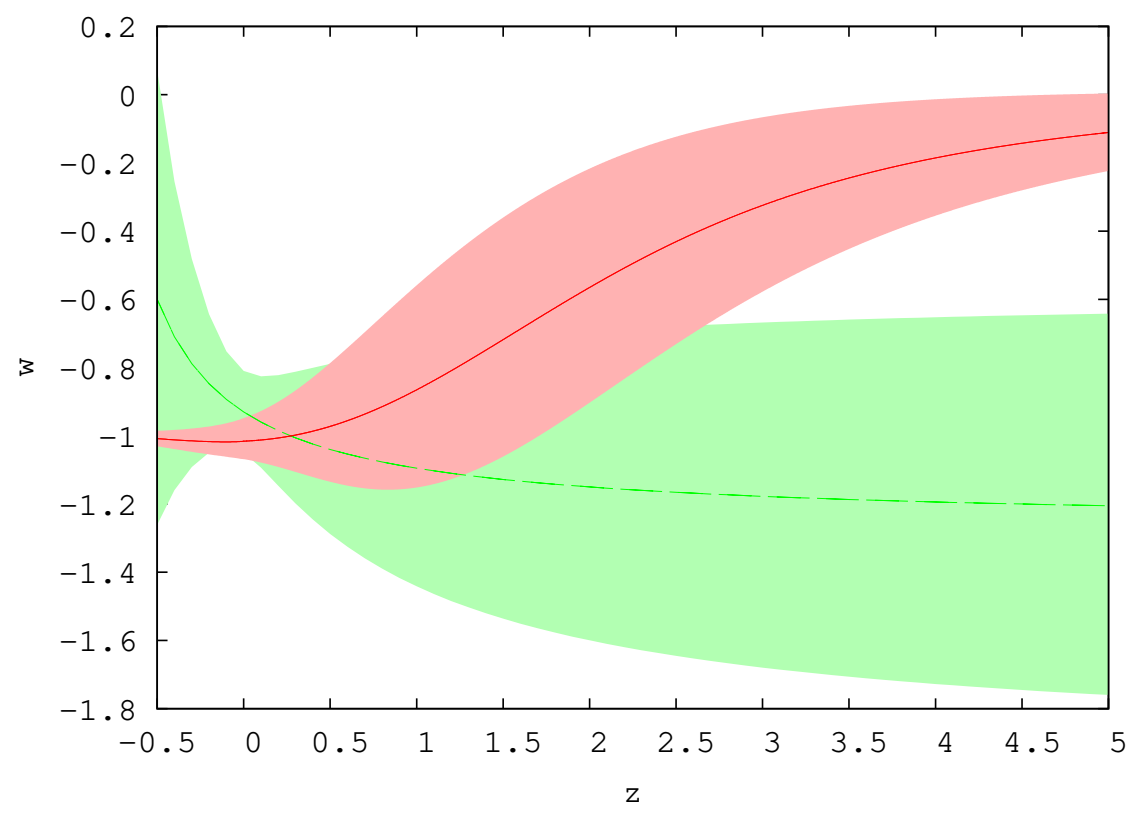

Figure 3. EoS parameters vs. redshift. The shaded area shows the $1 \sigma$ confidence region. The solid (red) and the dashed (green) lines are used for the best fit of parametrization I (Eq. (11)) and for the CPL parameterization, Eq. (14), both with $\Omega_{M 0}=0.27 \pm 0.03$. For the CPL parameterization the values $w_{0}=-0.93 \pm 0.12$ and $w_{1}=-0.38_{-0.65}^{+0.66}$ obtained by Komatsu et al. [24] were used.

Left panel of Fig. 4 shows the evolution of $q$ for the best fit value of parametrization II (with its $1 \sigma$ confidence region) and the $\Lambda$ CDM model obtained by Komatsu et al. [24]. The right panel depicts the evolution of the Hubble function versus redshift in the interval $0 \leq z \leq 3$

\begin{tabular}{||l||lllll||}
\hline \hline Data sets & $\chi_{S N}^{2}$ & $\chi_{B A O / C M B}^{2}$ & $\chi_{H}^{2}$ & $\chi_{\text {tot }}^{2}$ & $\chi_{\text {tot }}^{2} /$ dof \\
\hline \hline Union2+CMB/BAO+Hubble & 542.7 & 2.7 & 17.7 & 563.1 & 0.96 \\
\hline \hline
\end{tabular}

Table III. Same as Table II but for parametrization II, Eq. (15)

Figure 5 shows the $1 \sigma$ and $2 \sigma$ contour plots of the pair $\left(q_{1}, q_{2}\right)$ (left panel), and $\left(H_{0}, q_{0}\right)$ (right panel). Note that $q_{0}$ results more degenerate than in the previous parametrization (as well as in the next one). This arises because -as direct inspection shows- in the other two parametrizations $q_{0}$ depends on just one free parameter, $q_{1}$, while in this parametrization it depends on both, $q_{1}$ and $q_{2}$. 

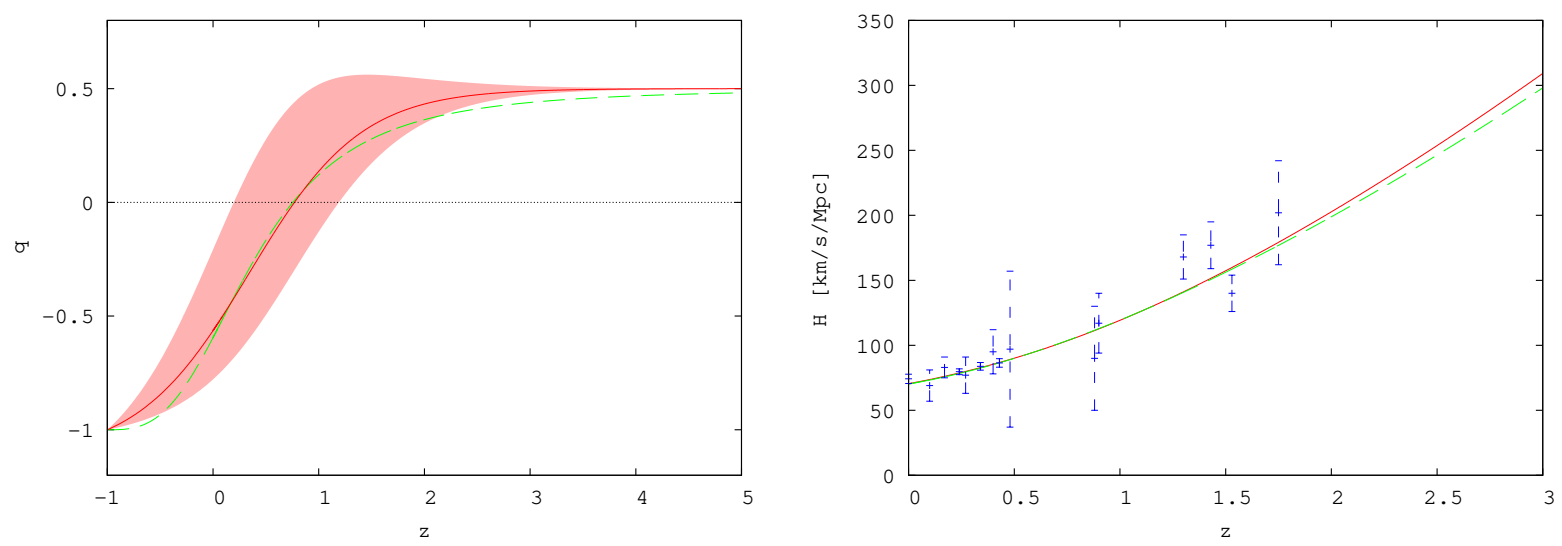

Figure 4. Same as Fig. 1 but for parametrization II, Eq. (15).
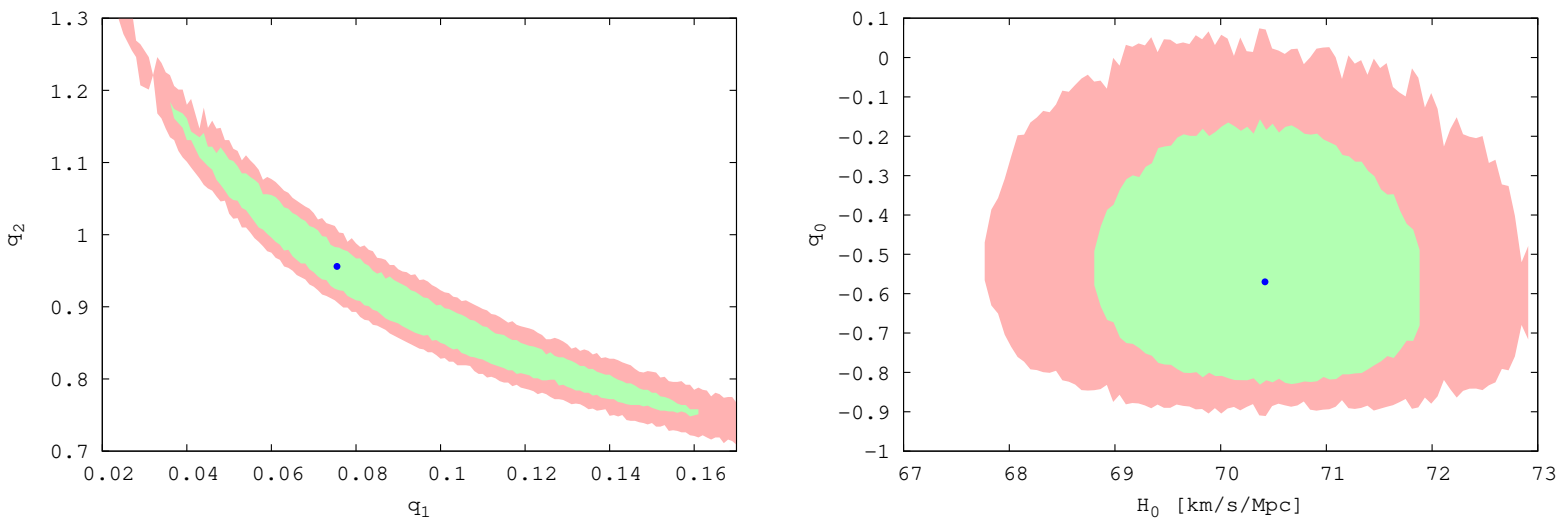

Figure 5. Same as Fig. 2 but for parametrization II, Eq. (15).

Considering the effective EoS parameter (13), as in the previous section, we obtain after linearization $w_{0}=-0.97_{-0.21}^{+0.33}$ and $w_{1}=-0.15_{-0.47}^{+0.70}$. As Fig. 6 shows, the evolution of the said effective EoS and the CPL in an extended redshift interval is similar to the previous one but with the $1 \sigma$ uncertainty interval significantly wider.

\section{Parametrization III}

The previous parametrization presents the inconvenience of a significant uncertainty in $q_{0}$ since it depends on the two free parameters. The following parametrization

$$
q(z)=-\frac{1}{4}+\frac{3}{4} \frac{q_{1} e^{q_{2} \frac{z}{\sqrt{1+z}}}-e^{-q_{2} \frac{z}{\sqrt{1+z}}}}{q_{1} e^{q_{2} \frac{z}{\sqrt{1+z}}}+e^{-q_{2} \frac{z}{\sqrt{1+z}}}} .
$$




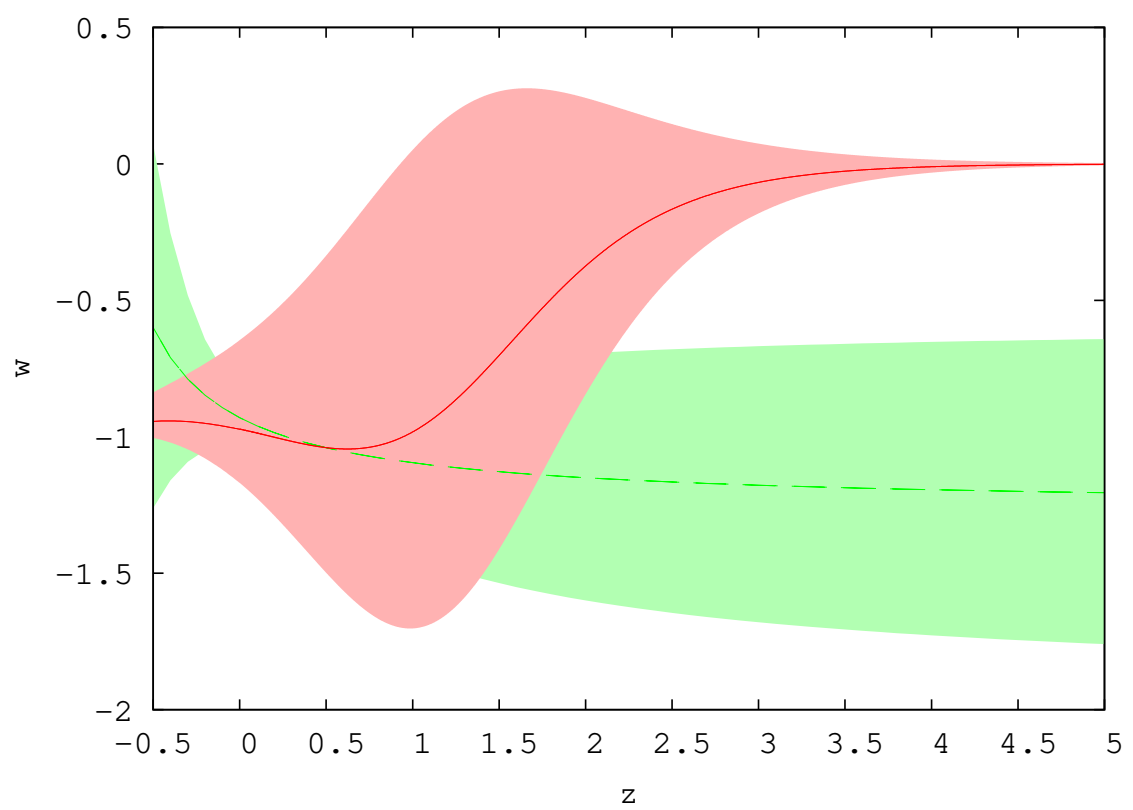

Figure 6. EoS parameters vs. redshift. The shaded area shows the $1 \sigma$ confidence region. The solid (red) and the dashed (green) lines are used for the best fit of parametrization I (Eq. (11)) and the CPL parametrization, Eq. (14), respectively, both assuming $\Omega_{M 0}=0.27 \pm 0.03$. For the CPL parametrization the values $w_{0}=-0.93 \pm 0.12$ and $w_{1}=-0.38_{-0.65}^{+0.66}$ obtained by Komatsu et al. [24] were used.

avoids this as $q_{0}$ depends on $q_{1}$ only.

Again, $H(z)$ must be obtained numerically. Then, proceeding as in the two previous instances, we obtain $q_{1}=0.36_{-0.08}^{+0.07}, q_{2}=1.57_{-0.33}^{+0.27}$, and $H_{0}=70.5_{-1.6}^{+1.4} \mathrm{~km} / \mathrm{s} / \mathrm{Mpc}$. The $\chi^{2}$ values of the best the fit are indicated in table [V]. Figure [7 shows the evolution of the

\begin{tabular}{||l||lcccc||}
\hline \hline Data sets & $\chi_{S N}^{2}$ & $\chi_{B A O / C M B}^{2}$ & $\chi_{H}^{2}$ & $\chi_{\text {tot }}^{2}$ & $\chi_{\text {tot }}^{2} / d o f$ \\
\hline \hline Union2+CMB/BAO+Hubble & 542.6 & 1.7 & 17.9 & 563.2 & 0.96 \\
\hline \hline
\end{tabular}

Table IV. Same as Table II but for parametrization III, Eq. (16).

deceleration parameter for the best fit parametrization (with its $1 \sigma$ confidence region) and the $\Lambda$ CDM model as determined the WMAP team [24] (left panel), and the evolution of the Hubble function (right panel).

Fig. 8 shows the $1 \sigma$ and $2 \sigma$ contour plots of the pairs $\left(q_{1}, q_{2}\right)$ (left panel) and $\left(H_{0}, q_{0}\right)$ (right panel). 

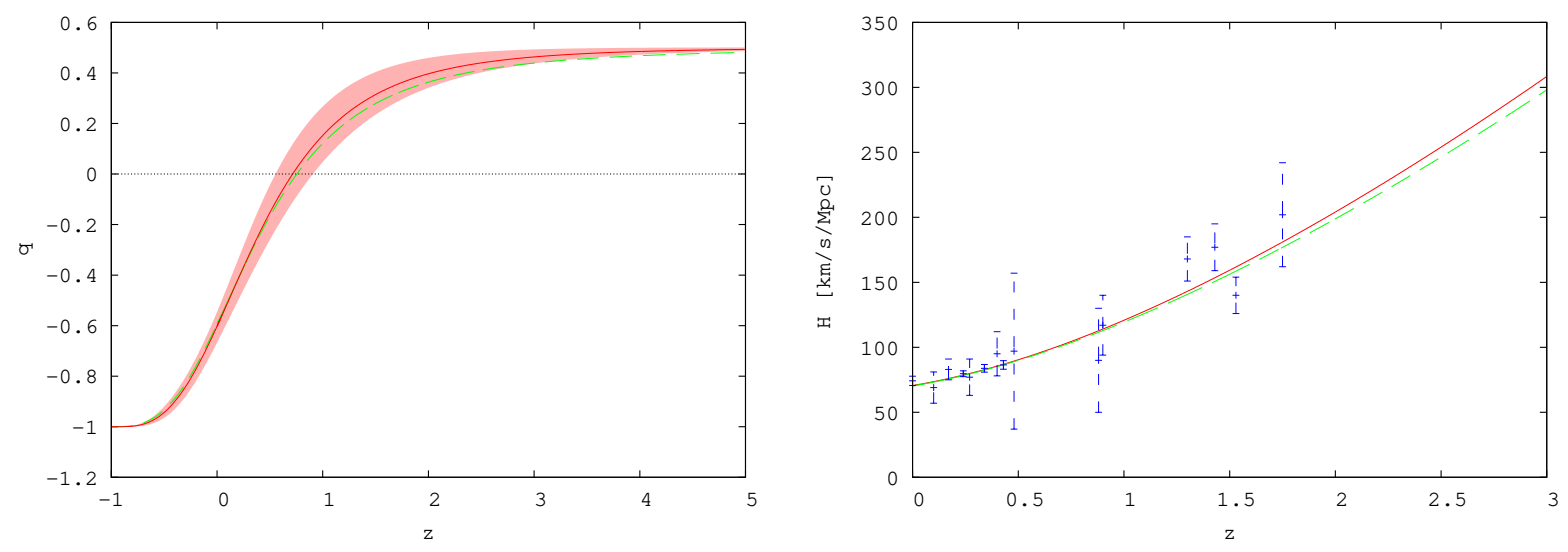

Figure 7. Same as Fig. 1 but for parametrization III, Eq. (16).
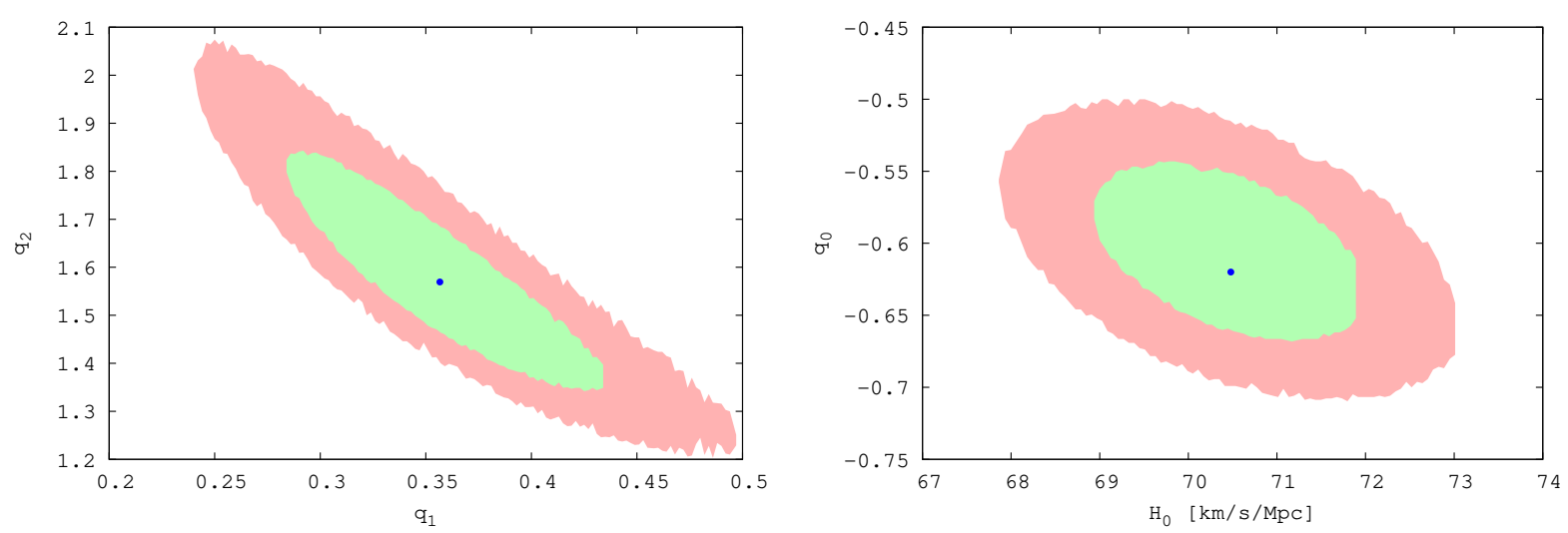

Figure 8. Same as Fig. 2 but for parametrization III, Eq. (16).

As for the effective EoS parameter (13), proceeding as in the previous subsections, we obtain $w_{0}=-1.01 \pm 0.06$ and $w_{1}=0.03 \pm 0.16$. Again, the evolution of the said effective EoS and the CPL in an extended redshift interval is rather similar to the one in Fig. 3, thereby, we do not feel it necessary to show it here.

\section{Discussion}

Figure 9 compares the parametrizations. All three yield rather similar results being really close between one another from the statistical standpoint $\left(\chi^{2} / d o f=0.96\right.$ for all of them). However, parametrization II looks somewhat less favored than the other two because of the 
noticeably wider $1 \sigma$ region of $q$ vs. $z$, as seen in the left panel of Fig. 4 .

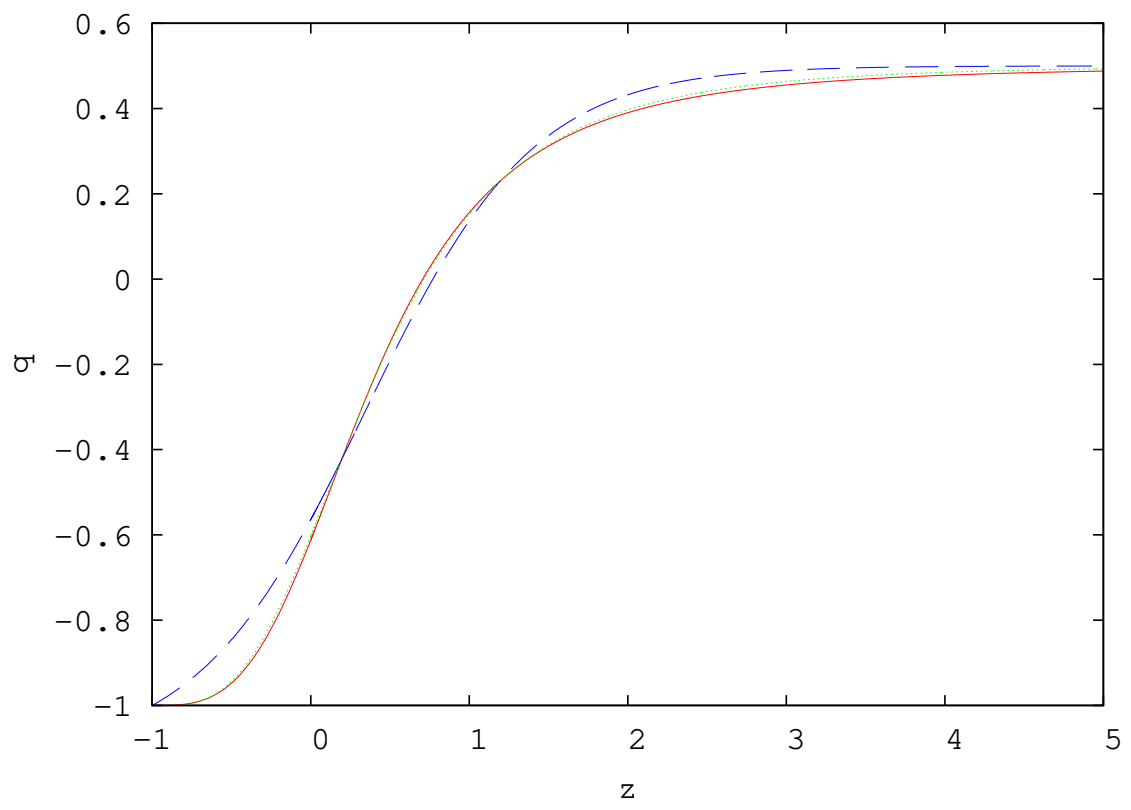

Figure 9. Deceleration parameters vs. redshift. Solid (red), long dashed (blue) and short dashed (green) lines are for parametrizations I, II and III respectively. The graphs of parameterizations I and III practically overlap each other.

All of them are consistent with a present stage of accelerated expansion, never to end or slow down. Further, the best fit plots of $H(z)$ and $q(z)$ are alike the corresponding plots of the $\Lambda$ CDM model as determined by Komatsu et al. [24]. From Table V] we learn that all $H_{0}$ best fit values are within $1 \sigma$ of each other and consistent with the $H_{0}$ value reported in [24]. The same holds true for the best fits of the age of the Universe (given by $\left.t_{0}=\int_{0}^{\infty} d z /[(1+z) H(z)]\right), q_{0}$ and the redshift, $z_{t}$, at which the transition decelerationacceleration occurred (i.e., $q\left(z_{t}\right)=0$ ), though the central values of the latter are not so close between each other as the corresponding values of the other two parameters. At any rate, the three of them are consistent with the $z_{t} \approx 0.5$ value obtained by $\mathrm{Wu}$ et al. using the history of the strong energy condition [35], as well as with the findings of Riess et al. [36], Cunha and Lima [5], and Lu et al. [37].

As table $\left[\right.$ shows, the values predicted for Hubble's constant, $H_{0}$, by the three parametrizations are within $1 \sigma$ between one another and with the value predicted by the $\Lambda$ CDM model that best fit identical sets of observational data. This is also true for $t_{0}, q_{0}$ and $z_{t}$. 


\begin{tabular}{||l||cccc||}
\hline \hline & Param. I & Param. II & Param. III & \multicolumn{1}{c||}{$\Lambda \mathrm{CDM}$} \\
\hline \hline$H_{0}$ & $70.5_{-1.6}^{+1.5}$ & $70.4 \pm 1.6$ & $70.5_{-1.6}^{+1.4}$ & $70.2 \pm 1.4$ \\
\hline$t_{0}$ & $13.6 \pm 0.5$ & $13.7 \pm 0.4$ & $13.6 \pm 0.2$ & $13.4 \pm 0.1$ \\
\hline$q_{0}$ & $-0.61_{-0.07}^{+0.06}$ & $-0.56_{-0.22}^{+0.35}$ & $-0.60 \pm 0.06$ & $-0.60 \pm 0.03$ \\
\hline$z_{t}$ & $0.71_{-0.17}^{+0.14}$ & $0.77_{-0.57}^{+0.52}$ & $0.72_{-0.21}^{+0.27}$ & $0.76 \pm 0.05$ \\
\hline \hline
\end{tabular}

Table V. Hubble's constant, $H_{0}$ (in $\mathrm{km} / \mathrm{s} / \mathrm{Mpc}$ ), the age of the Universe, $t_{0}$ (in Gyr), the deceleration parameter, $q_{0}$, and the redshift, $z_{t}$, of the transition deceleration-acceleration for the three parametrizations, and the flat $\Lambda \mathrm{CDM}$ model fitted to the same data sets.

A direct and model-independent determination of $q(z)$ in the redshift interval $0 \leq z \leq 1$ was carried out by Daly and coworkers [38] who applied the expression [39]

$$
q(z)=-1-(1+z)\left[\frac{d^{2} y / d z^{2}}{d y / d z}+\frac{\Omega_{k 0} y d y / d z}{1+\Omega_{k 0} y^{2}}\right]
$$

to the 192 SN I data points of Davis et al. [40] and 30 radiogalaxy data points of Daly et al. [41] -see Fig. 10 in [38]. In (17) $y(z)=H_{0}\left(a_{0} r\right)$ is the dimensionless coordinate distance, $r$ the radial coordinate of the FLRW metric, and $\Omega_{k 0}=-k /\left(H_{0} a_{0}\right)^{2}$. Notice that it just assumes the FLRW metric; i.e., it holds regardless the energy components of the Universe or the specific theory of gravity adopted.

Figure 10 shows the best fit graphs of $q(z)$ of each parametrization superimposed to experimental results of Daly et al. [38]. As it is seen, these lines fall within the $1 \sigma$ region of $q(z)$ as determined in Ref. [38].

One may ask as to what extent the imposing of the thermodynamic-based, far future, constraint $q(z=-1)=-1$ biases the parametrizations toward the $\Lambda$ CDM model. We have studied this by letting the value of $q(z=-1)$ as an additional free-parameter and fitting it using solely the observational data. The results are: $q(z=-1)=-0.65_{-0.50}^{+0.21}$, $q(z=-1)=-1.1_{-1.7}^{+0.2}$, and $q(z=-1)=-0.82_{-0.4}^{+0.07}$ for parametrizations I, II and III, respectively. Except for the second one, the quintessence cold dark matter (CDM) model is somewhat preferred but, in all the cases, the physically motivated choice $q(z=-1)=-1$ results compatible within $1 \sigma$. The drawback of letting $q(z=-1)$ free, aside from violating thermodynamics, is that the other two free-parameters present a wide degeneracy.

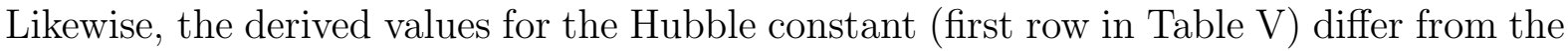
recently obtained by Riess et al., $H_{0}=74.2 \pm 3.6 \mathrm{~km} / \mathrm{s} / \mathrm{Mpc}$, [26], who used 240 Cepheids 


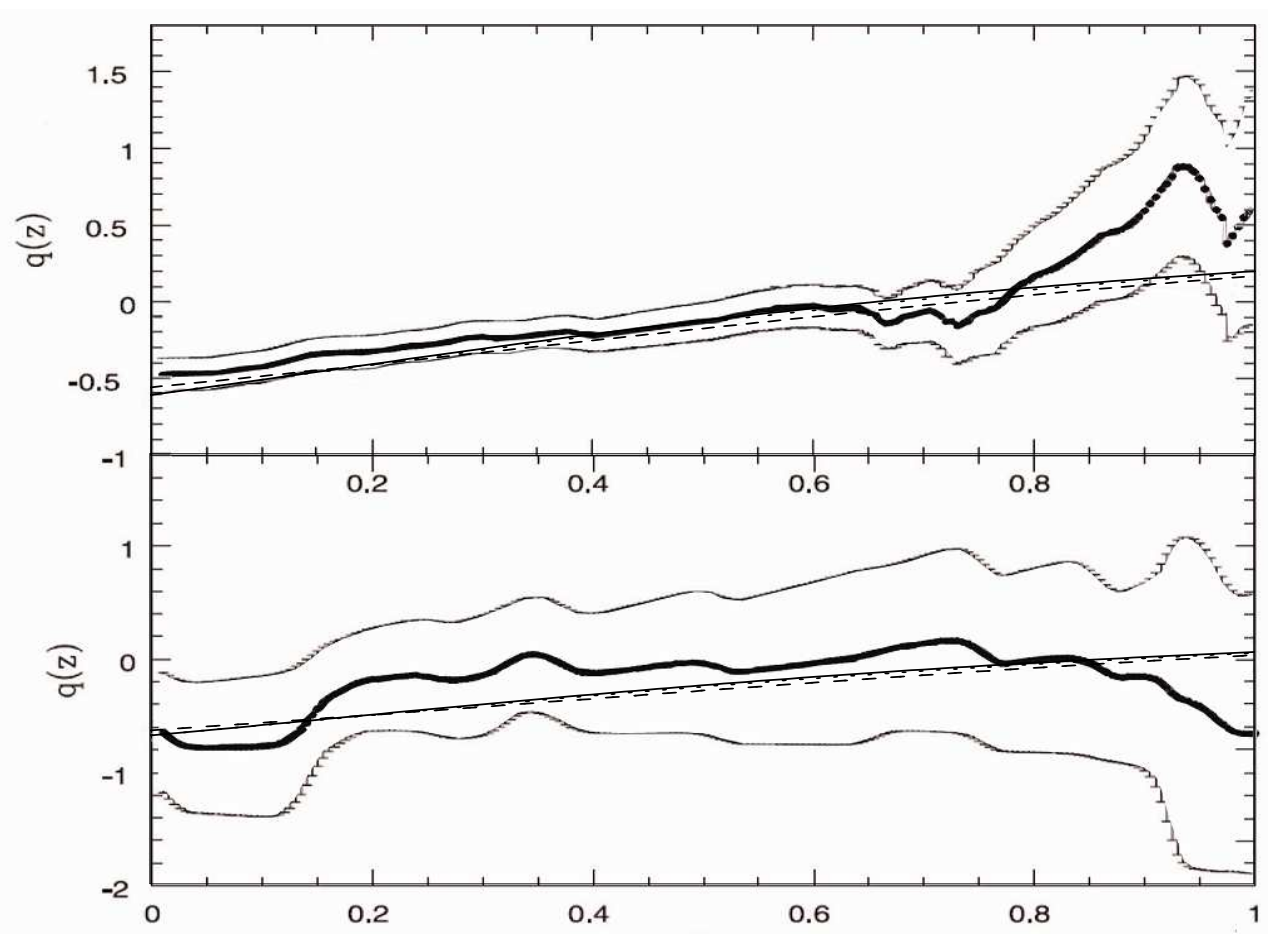

Figure 10. Top panel: observational constraints, at $1 \sigma$ confidence level, on the deceleration parameter versus redshift, obtained by Daly et al. using the combined sample 192 supernovae and 30 radio galaxies (top panel of Fig. 10 in [38]); the thick solid line corresponds to the central experimental value of $q(z)$. Solid, dashed, and dot-dashed lines correspond to parametrizations I (Eq. (11)), II (Eq. (15)) and III (Eq. (16)), respectively. Bottom panel: The same as the top panel except that the observational constraints on $q(z)$ were obtained solely from the sample of 30 radio galaxies (bottom panel of Fig. 10 in [38]). In both panels parametrizations I and III practically overlap each other.

variables at $z<0.1$, by about $6 \%$ (but they all agree with the latter at $1 \sigma$ ). We have considered this by repeating the analysis of subsection IIIC but this time leaving aside the mentioned value of Riess et al. The results now are: $H_{0}=70.0_{-1.5}^{+1.7}, H_{0}=70.0_{-1.6}^{+1.5}$, and $H_{0}=70.1_{-1.6}^{+1.6} \mathrm{Km} / \mathrm{s} / \mathrm{Mpc}$ for parametrizations I, II and III, respectively. Thus, both sets of results are essentially coincident (they differ by less than 1\%). They also agree very well with 
the Hubble constant value observationally derived by Komatsu et al., $70.4 \pm 2.5 \mathrm{~km} / \mathrm{s} / \mathrm{Mpc}$ [24], using WMAP 7-year data. So, while there is a significant difference between the Hubble constant value of Riess et al. and ours, it is not a substantial one; after all, they agree at $1 \sigma$ confidence level. At any rate, the root of the discrepancy may be rightly traced at the difference in methods employed. While Riess et al. essentially used astrophysical data, we (as well as Komatsu et al.) resorted to cosmological data instead.

\section{CONCLUDING REMARKS}

In this paper we proposed three different two-parameter parametrizations of $q(z)$ valid from the matter era $(z \gg 1)$ up to the infinite future $(z=-1)$, modulo $H(z)>0$. These rest in the following hypotheses: $(i)$ at cosmological scales the Universe is homogeneous and isotropic, thereby well described by the FLRW metric; (ii) in the matter dominated era $q=0.5 ;($ iii $)$ at least at late times the entropy of the Universe is dominated by the entropy of the apparent horizon. The second an third hypotheses furnish two fixed points (at $z \gg 1$ and $z=-1$, respectively), thereby drastically reducing the ample latitude one faces in parameterizing $q(z)$. By smoothly interpolating between these two points one can obtain useful parametrizations, but with shrunk arbitrariness.

Except for the existence of a matter dominated era at early times, the parametrizations are independent on any specific cosmological model; and, on the other hand, they are flexible enough to accommodate many homogeneous and isotropic models. We constrained the free parameters with the latest observational data (SN Ia, BAO, CMB, and $H(z)$ ). Accordingly

if to accommodate a given cosmological model the free parameters, $q_{1}$ and $q_{2}$, in the three parametrizations should take values widely apart from their respective best fits (which are consistent within $1 \sigma$ with the flat $\Lambda$ CDM model), we may confidently discard the said model.

Thermodynamics in spatially flat $(k=0)$ FLRW universes demands that $q(z=-1)=$ -1. This provides us with an additional and very useful fixed point to parametrize $q(z)$ in a model independent manner. Note that in the absence of a physically-based guidance to figure out the value of the deceleration parameter at $z=-1$ one is led to choose some or other random value. By contrast, in our case we have taken $q(z=-1)=-1$ on solid thermodynamic grounds.

Albeit we have considered just the particular set of spatially flat FLRW universes, it is 
not a big restriction at all. Indeed, recalling that in the case of non-flat metrics the area of the apparent horizon is given by Eq. (11) it follows that $\mathcal{A}^{\prime}=\mathcal{A}^{2} /(2 \pi a)\left[H^{2}(1+q)+k a^{-2}\right]$. When $a \rightarrow \infty$ and $k=-1$, the last term on the right hand side is necessarily subdominant otherwise one would have $\mathcal{A}^{\prime}<0$, contrary to the second law. Hence the condition $\mathcal{A}^{\prime} \geq 0$ in that limit reduces to the one in flat space, namely, $q \geq-1$. In the positively curved case, and again in the same limit, one can assume that $H \propto a^{n}, n$ being some real number. As it can be straightforwardly checked, the aforementioned last term results, once more, subdominant provided that $n>-1$ which is the case of most realistic cosmologies. Thus, $(z=-1, q=-1)$ is an asymptotic fixed point not only for spatially flat universes but also for open universes and for a rather ample set of closed universes.

Our results suggest that from the era of matter domination onward $q$ decreases monotonously with expansion (i.e., $d q / d z>0$ ), the transition deceleration acceleration occurred at a redshift of about 0.7 , and that $q_{0} \simeq-0.6$. They do not support recent claims that the cosmic expansion is today reverting to a decelerated phase (i.e., that $\left.d q /\left.d z\right|_{0}<0\right)$ [34], [42]-[45]. On the contrary, they show overall consistency with the findings of [2]-[10], [38], [46], as well as with those of Serra et al., [47]. The latter authors showed that the equation of state of dark energy has not varied noticeably in the redshift interval $0 \leq z \leq 1$. 


\section{ACKNOWLEDGMENTS}

We are indebted to Bin Wang for useful advice and the anonymous referee for helpful comments. This work was partially supported from "Comisión Nacional de Ciencias y Tecnología" (Chile) through the FONDECYT Grant No. 1110230 (SdC and RH) and No. 1090613 (RH and SdC). D.P. acknowledges "FONDECYT-Concurso incentivo a la cooperión internacional" No. 1110230 and is grateful to the "Instituto de Física", where part of this work was done, for warm hospitality. Also I.D. and D.P. research was partially supported by the "Ministerio Español de Educación y Ciencia" under Grant No. FIS2009-13370-C02-01, and by the "Direcció de Recerca de la Generalitat" under Grant No. 2009SGR-00164.

[1] M. Li, X.D. Li, S. Wang, and Y. Wang, arXiv:1103.5870[astro-ph.CO].

[2] Ø. Elgarøy and T. Multamäki, JCAP 09(2006)002.

[3] C.A. Shapiro and M.S. Turner, Astrophys. J. $\underline{649}$, 563 (2006).

[4] Y. Gong and A. Wang, Phys. Rev. D $\underline{75}, 043520$ (2007).

[5] J.V. Cunha and J.A.S. Lima, Mon. Notices R. Astron. Soc. 390, 210 (2008).

[6] L. Xu and J. Lu, Mod. Phys. Lett. A $\underline{24}, 369$ (2009).

[7] B. Santos, J.C. Carvalho, and J.S. Alcaniz, arXiv:1009.2733.

[8] R. Nair, S. Jhingan, and D. Jain, JCAP 01(2012)018.

[9] E.E.O. Ishida, R.R.R. Reis, A.V. Toribio, and I. Waga, Astroparticle Physics 28, 547 (2008).

[10] R. Giostri, M. Vargas dos Santos, I. Waga, R.R.R. Reis, M.O. Calvão, and B.L. Lago, JCAP03 (2012) 027.

[11] H.B. Callen, Thermodynamics (J. Wiley, New York, 1960).

[12] C.A. Egan and C.H. Lineweaver, Astrophys. J. $\underline{710}$, 1825 (2010).

[13] D. Bak and S.J. Rey, Class. Quantum Grav. 17, L83 (2000).

[14] B. Wang, Y. Gong, and E. Abdalla, Phys. Rev. D. 74, 083520 (2006).

[15] N. Radicella and D. Pavón, Gen. Relativ. Grav. 44, 685 (2012).

[16] R. Amanullah et al. (The Supernova Cosmology Project), Astrophys. J. $\underline{716}, 712$ (2010).

[17] S. Nesseris and L. Perivolarapoulos, JCAP 01 (2007) 018.

[18] D.J. Eisenstein et al. (DSS Collaboration), Astrophys. J. $\underline{633}, 560$ (2005). 
[19] W.J. Percival et al., Mon. Not. R. Astron. Soc. 401, 2148 (2010).

[20] E. A. Kazin et al., Astrophys. J. $\underline{710}, 1444$ (2010).

[21] F. Beutler, C. Blake, M. Colles, D.H. Jones, L. Staveley-Smith, L. Campbell, Q. Parker, W. Saunders, and F. Watson, Mon. Not. R. Astron. Soc. $\underline{416}, 3017$ (2011).

[22] A. Blake et al., Mon. Not. R. Astron. Soc. $\underline{418}, 1707$ (2011).

[23] D. J. Eisenstein and W. Hu, Astrophys. J. $\underline{496}, 605$ (1998).

[24] E. Komatsu et al., Astrophys. J. Suppl. Ser. 192, 18 (2011).

[25] J. Sollerman et al., Astrophys. J. $\underline{703}$, 1374 (2009).

[26] A. Riess et al., Astrophys. J. $\underline{699}, 539$ (2009).

[27] E. Gaztañaga, A. Cabré, and L. Hui, Mon. Not. R. Astron. Soc. 399, 1663 (2009).

[28] J. Simon, L. Verde, and R. Jiménez, Phys. Rev. D 1ㅡ, 123001 (2005).

[29] D. Stern, R. Jiménez, L. Verde, M. Kamionkowski, and S.A. Stanford, JCAP02 (2010) 008.

[30] C. Blake et al., "The WiggleZ Dark Energy Survey: Joint measurements of the expansion and growth history at $z<1 "$, arXiv:1204.3674.

[31] M. Chevallier and D. Polarski, Int. J. Mod. Phys. D 무, 213 (2001).

[32] E.V. Linder, Phys. Rev. Letters 무, 091301 (2003).

[33] Z. Li, P. Wu, and H. Yu, Phys. Letters B $\underline{695}, 1$ (2011).

[34] V. Cardenas, and M. Rivera, Phys. Lett. B 1ㅡ, 251 (2012).

[35] C.-J. Wu, C. Ma, and T.-J. Zhang, Astrophys. J. 753, 97 (2012).

[36] A.G. Riess et al., Astrophys. J. $\underline{659}, 98$ (2007).

[37] J. Lu, L. Xu, and M. Liu, Phys. Letters B $\underline{699}, 246$ (2011).

[38] R.A. Daly, S.G. Djorgovski, K.A. Freeman, M.P. Mory, C.P. O’Dea, P. Kharb, and S. Baum, Astrophys. J. 677, 1 (2008).

[39] R.A. Daly Advances in Space Research 포, 116 (2005).

[40] T.M. Davis et al., Astrophys. J. $\underline{666}, 716$ (2007).

[41] R.A. Daly, M.P. Mory, C.P. O’Dea, P. Kharb, S. Baum, E.J. Guerra, and S. Djorgovski, Astrophys. J. 691, 1058 (2009).

[42] A. Shafieloo, V. Sahni and A.A. Starobinsky, Phys. Rev. D $\underline{80}, 101301$ (2009).

[43] Z. Li, P. Wu, and H. Yu, Phys. Lett. B $\underline{695}, 1$ (2011).

[44] A.C.C. Guimarães and J.A.S. Lima, Class. Quantum Grav. 28, 125026 (2011).

[45] C. Zuñiga-Vargas, W.S. Hipólito-Ricaldi, and W. Zimdahl, JCAP 04 (2012) 032. 
[46] A.C.C. Guimarães, J.V. Cunha, and J.A.S. Lima, JCAP 10(2009)010.

[47] P. Serra, A. Cooray, D.E. Holz, A. Melchiorri, S. Pandolfi, and D. Sarkar, Phys. Rev. D. $\underline{\text { 80}}$, 121302(R) (2009). 